\title{
Identification of up-regulated genes during zearalenone biosynthesis in Fusarium
}

\author{
Erik Lysøe • Karen R. Bone • Sonja S. Klemsdal
}

Received: 25 November 2007 / Accepted: 7 April 2008/Published online: 26 April 2008

(C) KNPV 2008

\begin{abstract}
The Fusarium genus includes devastating plant pathogenic fungi that cause diseases in cereals around the world. They produce several mycotoxins, including the estrogenic compound zearalenone. To better understand the molecular mechanisms determining zearalenone production, we performed differential display RT-PCR under conditions where Fusarium graminearum and F. culmorum produced high amounts of zearalenone. We found 133 expressed sequence tags (ESTs) and 54 of these were considered to be up-regulated during high zearalenone production. Several of the ESTs were confirmed to be up-regulated by real-time qPCR, but none showed any significant down-regulation in the zearalenone
\end{abstract}

Electronic supplementary material The online version of this article (doi:10.1007/s10658-008-9318-x) contains

supplementary material, which is available to authorized users.

E. Lysøe $\cdot$ K. R. Bone $\cdot$ S. S. Klemsdal $(\square)$

Plant Health and Plant Protection Division,

Bioforsk-Norwegian Institute for Agricultural

and Environmental Research,

Høgskoleveien 7,

N-1432 Ås, Norway

e-mail: sonja.klemsdal@bioforsk.no

E. Lysøe

Department of Chemistry,

Biotechnology and Food Science,

Norwegian University of Life Sciences,

P.O. Box 5003, N-1432 Ås, Norway negative mutant $\triangle \mathrm{PKS} 4-\mathrm{T} 9$, or were similar to typical gene expression patterns of previously described zearalenone-related genes. Some of the up-regulated ESTs were similar to genes involved in secondary metabolite production, lipid metabolism, transcriptional activation, provision of precursors, signal transduction, transport or detoxification. Several of the ESTs were also located adjacent to one another in the genome and therefore might represent genes involved in the same biosynthetic pathway. Members of six such putative pathways could be found. All sequences were compared to the MIPS F. graminearum Genome Database to verify autocalled gene predictions experimentally and to introduce new exons and gene structures.

Keywords Differential display

Expressed sequence tags · Gene expression ·

Mycotoxin $\cdot$ Real-time qPCR $\cdot$ ZON

\begin{tabular}{|c|c|}
\hline \multicolumn{2}{|c|}{ Abbreviations } \\
\hline DDRT-PCR & Differential display RT-PCR \\
\hline$(\mathrm{ESTs})$ & Expressed sequence tags \\
\hline (FGDB) & $\begin{array}{l}\text { Fusarium graminearum Genome } \\
\text { Database }\end{array}$ \\
\hline (FHB) & Fusarium Head Blight \\
\hline (HMG) & Hydroxymethylglutaryl \\
\hline (PKS) & Polyketide synthase \\
\hline$(\mathrm{ZON})$ & Zearalenone \\
\hline
\end{tabular}




\section{Introduction}

ZON is a mycotoxin produced by several Fusarium species and has been associated with estrogenic syndromes in humans and animals. Fusarium graminearum [teleomorph Gibberella zeae] and F. culmorum are the best known ZON producers and causative agents of FHB, also known as scab, on small-grain cereals (Parry et al. 1995). ZON can contaminate grains in the field when environmental conditions favour fungal infection, and levels can increase dramatically if storage conditions are favourable for fungal growth. It is believed that high humidity and low temperature promote $\mathrm{ZON}$ production (Coulombe 1993). ZON is a polyketide, a thermostable metabolite unaffected by processing techniques (Lauren and Smith 2001). Other common Fusarium mycotoxins are trichothecenes and fumonisins. ZON produced by $F$. graminearum and $F$. culmorum and is frequently found in Fusarium-infected cereals in Europe (Bottalico and Perrone 2002). The economic damage and mycotoxin contamination of agricultural products caused by Fusarium spp. can be considerable in years when weather conditions favour development of FHB (http://www.scabusa.org/). The European Union has therefore issued directives that set maximum allowable levels for ZON and other Fusarium toxins in food and feed (Commission Regulation (EC) No 856/2005).

The genome sequence of $F$. graminearum has been determined by the Broad Institute (http://www.broad. mit.edu) and annotated by the MIPS FGDB (Guldener et al. 2006a). We have previously reported that the PKS gene PKS4 is essential for ZON production in $F$. graminearum (Lysoe et al. 2006). Three other genes, PKS13, a gene similar to alcohol oxidase (FG12056) and the putative transcription factor (FG02398) have also been found to be required for ZON production (Gaffoor and Trail 2006; Kim et al. 2005). These four ZON genes lie clustered in the genome. Additional genes outside this cluster involved in ZON biosynthesis have not yet been identified. Previous reports have shown that ZON has neither an effect on the pathogenicity of $F$. graminearum on barley seedlings (Lysoe et al. 2006) nor the infection of barley or wheat heads (Gaffoor et al. 2005; Kim et al. 2005), and the exact function of $\mathrm{ZON}$ in the fungus is not known. In this study we have used the method of DDRT-PCR to identify up-regulated genes during $\mathrm{ZON}$ production.

\section{Materials and methods}

Strains and culture conditions

F. graminearum 1104-14, 1194-15, $1166-9$ and F. culmorum 1098-6 (Langseth et al. 1998) were obtained from the Fusarium collection at the National Veterinary Institute of Norway. These strains are high $\mathrm{ZON}$ producers on sterile rice. The $\mathrm{ZON}$ negative mutant $\triangle$ PKS4-T9 was derived from 1104-14 via homologous recombination and replacement of the central part of PKS4 with hygB (Lysoe et al. 2006). For RNA extraction and analysis of ZON production in DDRT-PCR experiments, strains were cultivated on sterile rice at $25^{\circ} \mathrm{C}$ in the dark for up to 2 weeks, or with an additional cultivation at $12^{\circ} \mathrm{C}$ for up to 6 weeks. Twenty grams of rice and $20 \mathrm{ml}$ of milliQ water were mixed in $500 \mathrm{ml}$ Erlenmeyer flasks, left at room temperature for $1 \mathrm{~h}$ and then autoclaved at $121^{\circ} \mathrm{C}$ for $1 \mathrm{~h}$. The flasks were inoculated with $1 \times 10^{6}$ spores. We sampled the rice for the weekly analysis from different flasks for each specific condition, with a minimum of three replicate flasks per time point. Samples were analyzed for ZON production with ELISA (EZ-Quant ${ }^{\mathrm{TM}}$ Zearalenone Test Kit, Diagnostix, Canada) after 1, 2, 3 and 6 weeks. For the real-time gene expression studies and HPLC analysis of ZON production, the fungi were harvested after 4, 7, 11 and 14 days of growth at $25^{\circ} \mathrm{C}$ in the dark on sterile rice.

\section{DDRT-PCR experiments}

RNA was purified from $50 \mathrm{mg}$ fungal biomass using the RNeasy Plant Mini Kit (Qiagen, CA, USA), treated with RNase-free DNase I (Ambion, TX, USA), and reverse transcribed using Advantage ${ }^{\circledR}$ cDNA PCR Kit \& Polymerase Mix (Clontech, CA, USA). The resulting cDNA was used in DDRT-PCR with the Delta ${ }^{\mathrm{TM}}$ Differential Display Kit (Clontech) according to the manufacturer's recommendations, using their primers as well as the additional primers listed in Table S1. PCR was performed on a GeneAmp PCR System 9700 (Applied Biosystems, CA, USA) and the fragments were labelled with $[\alpha-33 \mathrm{P}] \mathrm{dATP}$ (Amersham Biosciences, Uppsala, Sweden).

Electrophoresis $(70 \mathrm{~W}$, constant power) was performed with an EC600 power supply (EC Apparatus Corporation, FL, USA) and the products were separated on $5 \%$ polyacrylamide/8M Urea gels $(0.2 \mathrm{~mm})$ in $0.5 \times$ 
TBE buffer. Gels were pre-run for 30 min before loading the samples. After electrophoresis the gel was dried on Whatman chromatography filter paper at $75^{\circ} \mathrm{C}$ for $1 \mathrm{~h}$. The dried gel was exposed to BioMax MR film (Kodak, NY, USA) overnight at $-80^{\circ} \mathrm{C}$. The position of the film on the dried gel was marked and 72 differentially expressed bands were excised from the gel with a clean scalpel and placed in separate $0.5 \mathrm{ml}$ tubes. Sterile $\mathrm{dH}_{2} \mathrm{O}$ $(40 \mu \mathrm{l})$ was added and the samples heated at $100^{\circ} \mathrm{C}$ for $5 \mathrm{~min}$. The filter paper was then removed and $7 \mu \mathrm{l}$ of the resulting liquid was used in a $50 \mu \mathrm{l} \mathrm{PCR}$ with the same DDRT-PCR primers for reamplification of the differentially expressed bands. The PCR products were assessed on a $0.8 \%$ agarose gel.

Sequence analysis

The DDRT-PCR fragments were cloned (TOPO TA Cloning, Invitrogen, CA, USA) and 10 clones per fragment were sequenced using the ABI PRISM ${ }^{\circledR}$ dGTP BigDye ${ }^{\circledR}$ Terminator Cycle Sequencing Kit (Applied Biosystems) with a 310 Genetic analyzer (Applied Biosystems). Standard BLAST analysis was performed on the resulting sequences using the $F$. graminearum genome sequence, the COGEME phytopathogen EST database (Soanes and Talbot 2006) and GenBank. The F. graminearum gene names used here are according to MIPS FGDB.

\section{Relative gene expression}

The F. graminearum isolates $1104-14,1166-9$ and the ZON negative mutant $\triangle$ PKS4-T9 were used in the gene expression study. Total RNA was extracted from $50 \mathrm{mg}$ rice culture using the Qiagen RNeasy kit (Qiagen, Valencia, CA, USA). Total RNA was treated with the TURBO DNA-free ${ }^{\mathrm{TM}}$ kit (Ambion, Austin, TX, USA), and first strand cDNA synthesis was carried out using TaqMan ${ }^{\circledR}$ Reverse Transcription Reagents (Applied Biosystems, Foster City, CA, USA), according to the manufacturer's instructions. Priming was done with random hexamers. Relative expression of the genes was determined over time via real-time qPCR on an Applied Biosystems 7900HT instrument, with a standard 96-well block and the SYBR $^{\circledR}$ Green PCR Master Mix SYBR ${ }^{\circledR}$ (Applied Biosystems) and gene-specific primers (Table S1). Three biological replicates were used. The housekeeping genes $\beta$-tubulin (FG09530) (Reischer et al. 2004), translation elongation factor $1 \alpha$ (FG08811), and ubiquitin conjugating enzyme (FG10805) were shown to be stably expressed via the BestKeeper software (Pfaffl et al. 2004). The BestKeeper Index (BK) was used to normalize target gene data. The ratios of the cycle crossing point $\left(C_{\mathrm{T}}\right)$ values/BK values in the replicate runs were used to calculate the coefficient of variation, and indicated a high degree of reproducibility in the interrun data. The gene expression results for all genes and time points were determined relative to the same expression data, the gene similar to alcohol oxidase (FG12056) in the strain 1104-14 after 7 days growth. FG12056 is a gene in the ZON cluster (Kim et al. 2005) with low gene expression during the time frame of 4-14 days growth on sterile rice. This calibration was done to enable us to compare the expression patterns of these genes with patterns from known ZON-related genes (Lysoe et al., 2008, submitted). REST software (Pfaffl et al. 2002) was used to determine relative gene expression from cycle crossing point data and statistical significance using a pair-wise fixed reallocation randomization test.

\section{HPLC analysis of ZON}

The rice cultures used in the real-time experiments were freeze-dried and ground, and $0.1 \mathrm{~g}$ of the samples was extracted and prepared according to the method described by Smedsgaard (1997). The extract was separated by reverse phase chromatography on an Eclipse XDB-C18 column $(150 \times 4.6 \mathrm{~mm})$ with a mobile phase of $55 \%$ acetonitrile in water with $0.1 \%$ phosphoric acid. ZON was detected with a diode array detector at $\lambda=236 \mathrm{~nm}$ and a bandwidth of $4 \mathrm{~nm}$. Additional identification was done by collecting 2 UV spectra per second from 200 to $400 \mathrm{~nm}$ with a resolution of $2 \mathrm{~nm}$. The amount of $\mathrm{ZON}$ in the extracts was calculated against a linear calibration curve of ZON (Sigma-Aldrich, Z2125) in the range of $0.1-200 \mu \mathrm{g} \mathrm{ml}^{-1}$ which bracketed the samples.

\section{Results}

$\mathrm{ZON}$ production on sterile rice

To study differences in ZON production, three Fusarium strains were cultivated on sterile rice in the dark for up to 2 weeks at $25^{\circ} \mathrm{C}$, or with an additional 
cultivation at $12^{\circ} \mathrm{C}$ for up to 6 weeks (Fig. 1). The samples were analyzed for accumulated ZON after 1, 2, 3 and 6 weeks of cultivation. All three isolates produced only small amounts of ZON after 1 week. These were used as ZON minimum samples in the DDRT-PCR experiments. At later time points, there were great differences in $\mathrm{ZON}$ production between the two $F$. graminearum isolates. The $F$. graminearum 1104-14 showed a high quantity of accumulated ZON ( $\sim 800 \mathrm{ppm})$ after 2 weeks on $25^{\circ} \mathrm{C}$, and after one additional week with a temperature shift to $12^{\circ} \mathrm{C}$ the amount was significantly decreased to $\sim 200 \mathrm{ppm}$. After 6 weeks the accumulated ZON further increased to $\sim 1,120 \mathrm{ppm}$. Fusarium graminearum 1194-15 showed a relatively low amount of ZON after 2 weeks ( $\sim 100 \mathrm{ppm})$. The temperature shift here increased ZON production up to $\sim 750 \mathrm{ppm}$ after 3 weeks and only a slight reduction in ZON was seen after 6 weeks growth ( 600 ppm). Fusarium culmorum 1098-6 had a stable but low increase in accumulated ZON over time, from $11 \mathrm{ppm}$ (1 week) to $300 \mathrm{ppm}$ (6 weeks).

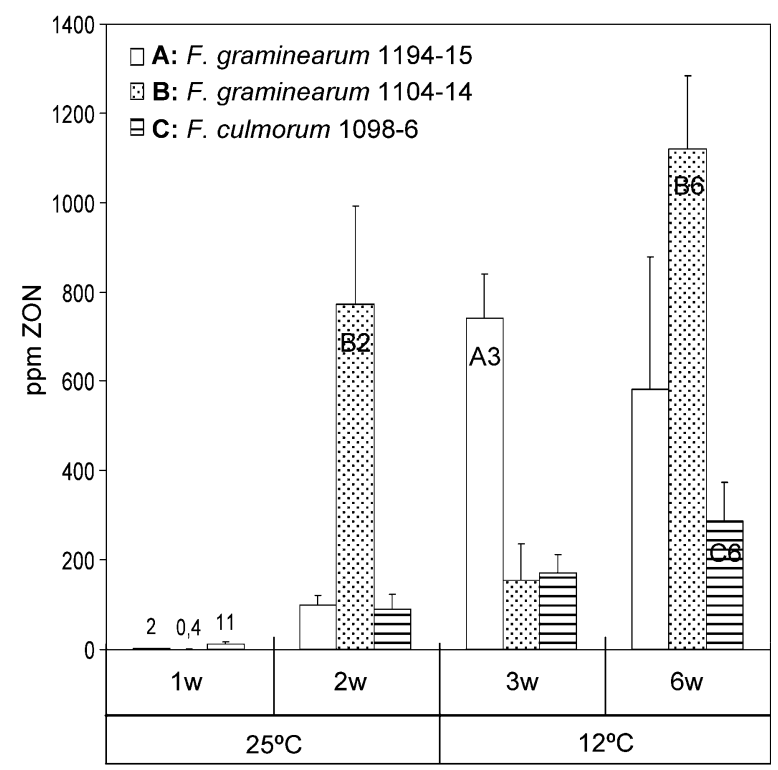

Fig. 1 Production of $\mathrm{ZON}$ after cultivation on sterile rice. Samples were harvested for $\mathrm{ZON}$ analysis after 1, 2, 3 and 6 weeks. The first 2 weeks at $25^{\circ} \mathrm{C}$ were followed by a temperature shift to $12^{\circ} \mathrm{C}$ for the next 4 weeks. Error bars indicate standard deviations. Samples A3, B2, B6 and C6 were used in DDRT-PCR as up-regulated ZON samples (maximum) and were compared to the three minimum samples after 1 week of cultivation (A1, B1 and $\mathrm{C} 1$ )

\section{DDRT-PCR analysis}

We performed DDRT-PCR on selected samples to identify and isolate up-regulated gene fragments during high ZON production. The ZON maximum samples used ranged from 300 to $1,120 \mathrm{ppm}$ and are shown as A3, B2, B6 and C6 in Fig. 1. These samples were compared to the $\mathrm{ZON}$ minimum samples after 1 week of cultivation, having ZON concentrations ranging from $0.4-11 \mathrm{ppm}$. The up-regulated bands during high ZON production were sequenced and subjected to various BLAST searches. The ESTs were submitted to GenBank (Tables 1, S2 and S3).

With the use of 41 primer combinations we obtained a total of 133 ESTs. Of these, 54 were characterized as putative up-regulated ESTs during high ZON production (Table 1). Several different criteria were used to minimize identification of false up-regulated bands. Of the 54 ESTs that we considered to be up-regulated, 18 were detected with several (up to seven) different oligo(dT) primers in combinations with the same arbitrary primer. These were products of combinations with only the arbitrary primer, and because of their redundancy we considered them likely to be up-regulated. We found that 18 of the up-regulated ESTs were found in two to three different strains and under different time points, adding confidence that the genes so identified were in fact up-regulated. Six groups of up-regulated ESTs corresponded to genes that were located close to one another on the same contigs (FG02666/FG02667/ FG02668, FG03081/FG03086, FG03184/FG03190/ FG03199, FG03735/FG03738, FG05928/FG05929 and FG07075/FG07076). These might therefore represent genes belonging to the same biosynthetic pathways. All these results, together with the finding of identical sequences of the clones originating from the same DNA band, contributed to minimize the likelihood of false positives. The 82 ESTs that were not considered to be up-regulated (the putative false positives) were found as a mixture of different ESTs from the same DDRT-PCR band.

Several of the ESTs found here could possibly be directly or indirectly connected to ZON production, but many are probably results of other processes. The culture are exposed to temperature decrease and ageing, and genes expressed early in culture when nutrients are relatively abundant are probably different from those expressed later when nutrients are 
Table 1 Putative up-regulated ESTs of Fusarium grown on sterile rice under high ZON-producing conditions

ESTs found in the published F. graminearum genome sequence

GenBank

accession

number

\begin{tabular}{|c|c|c|c|}
\hline C6 & 2 & FG01004-ext-Kinesin motor protein & DY588253 \\
\hline C6 & 1 & FG01101-Conserved hypothetical protein, TPR-like & ES316460 \\
\hline C6 & 2 & FG01417-Probable O-acetylhomoserine (thiol)-lyase & DY588265 \\
\hline B6 & 1 & No ORF hit, unknown & DY588276 \\
\hline A3/B6 & 1 & FG01584-ext - Conserved hypothetical protein & DY588287 \\
\hline $\mathrm{B} 2 / \mathrm{A} 3 / \mathrm{B} 6$ & 2 & No ORF hit—Similar to Gz47835827, E-115 & DY588295 \\
\hline $\mathrm{A} 3 / \mathrm{B} 6 / \mathrm{C} 6$ & 3 & FG01749—-Hypothetical protein, putative LIM domain protein (EAL87198) & DY588296 \\
\hline A3 & 1 & FG01826-Probable methylmalonate-semialdehyde dehydrogenase (acylating) & DY588297 \\
\hline A3/B6 & 1 & FG02666 — Related to acetylxylan esterase precursor & DY588298 \\
\hline C6 & 1 & FG02667-ext-Conserved hypothetical protein & DY588299 \\
\hline C6 & 1 & FG02668-Related to linoleate diol synthase & DY588254 \\
\hline C6 & 1 & FG02894 - Conserved hypothetical protein, putative oxidoreductase & DY588255 \\
\hline C6 & 1 & FG03081 - Conserved hypothetical protein, putative esterase & DY588256 \\
\hline $\mathrm{A} 3 / \mathrm{C} 6$ & 1 & FG03086 - Related to pisatin demethylase cytochrome P450 & DY588258 \\
\hline $\mathrm{A} 3 / \mathrm{B} 6 / \mathrm{C} 6$ & 1 & $\begin{array}{l}\text { FG03184-Hypothetical protein, putative RNA interference pathway protein } \\
\text { (Q09884) }\end{array}$ & DY588258 \\
\hline A3/B6 & 1 & $\begin{array}{l}\text { FG03190-ext-Hypothetical protein, resembles ethanolamine utilization protein eutQ } \\
\text { (EAL85854) }\end{array}$ & DY588259 \\
\hline B6 & 1 & FG03199-ext—Probable phenol 2-monooxygenase & DY588260 \\
\hline B6 & 1 & FG03735-Probable ABC1 transport protein & DY588261 \\
\hline B6 & 1 & FG03738-ext - Conserved hypothetical protein, putative esterase & DY588262 \\
\hline C6 & 6 & $\begin{array}{l}\text { FG05061-ext-Conserved hypothetical protein, a } \\
\text { putative pheromone precursor, alternative splicing }\end{array}$ & $\begin{array}{l}\text { DY588263 } \\
\text { DY588264 }\end{array}$ \\
\hline $\mathrm{A} 3 / \mathrm{B} 6 / \mathrm{C} 6$ & 3 & FG05095-Related to serine protease & DY588266 \\
\hline C6 & 1 & $\begin{array}{l}\text { No ORF hit-Similar to mitochondrial outer membrane protein involved in membrane } \\
\text { fission }(\mathrm{GzCon}[0585]), 9 \mathrm{E}-83\end{array}$ & DY588267 \\
\hline B6 & 4 & FG11310-ext-Putative protein [EST hit] & DY588268 \\
\hline B6 & 1 & FG05821-ext-Related to interferon-regulated resistance, GTP-binding protein & DY588269 \\
\hline B6 & 1 & FG05928-ext-Putative protein [EST hit] & DY588270 \\
\hline B6/A3 & 2 & FG05929-ext_-A possible extension. Calcium-binding EF-hand & DY588271 \\
\hline C6 & 1 & FG06070-Related to UTP6 - U3 snoRNP & DY588272 \\
\hline A3 & 1 & No ORF hit, unknown & DY588273 \\
\hline A3 & 1 & FG10387-ext—Probable DFG5 protein & DY588274 \\
\hline A3 & 1 & FG07075-ext_-Probable PYC2, pyruvate carboxylase 2 & DY763108 \\
\hline B6 & 1 & FG07076-ext-Cysteine-rich protein NFX-1 & DY588275 \\
\hline A3/B6 & 1 & FG07104—Probable peroxin-1 & DY588277 \\
\hline A3/B6 & 1 & FG07442 - Conserved hypothetical protein & DY588278 \\
\hline C6 & 1 & No ORF hit, unknown & DY588279 \\
\hline B6 & 1 & FG08245-ext-Hypothetical protein & DY588280 \\
\hline C6 & 1 & $\mathrm{Fg} 4 \mathrm{~A} 76$ - New orf, related to $\mathrm{SMC} 1$ protein (CAC18213) & DY588281 \\
\hline B6/C6 & 3 & FG08710-ext, probable septin aspE & DY588282 \\
\hline C6 & 1 & FG08811-ext-EF1A & DY588283 \\
\hline $\mathrm{A} 3 / \mathrm{B} 6 / \mathrm{C} 6$ & 2 & No ORF hit—Similar to Gibberella moniliformis cDNA (DR616112). 5E-33 & DY588284 \\
\hline All & 7 & FG09103-Related to gibberellin 20-oxidase & DY588286 \\
\hline $\mathrm{B} 2 / \mathrm{B} 6 / \mathrm{C} 6$ & 4 & FG09355-ext-Hypothetical protein & DY588288 \\
\hline B6 & 1 & FG09840-ext-Related to Gbp2p & DY588289 \\
\hline A3 & 1 & FG09913 - Related to dityrosine transporter & DY588290 \\
\hline C6 & 1 & FG09976 - Conserved hypothetical protein & DY588291 \\
\hline A3 & 1 & FG10084-Hypothetical protein & DY588292 \\
\hline B6 & 1 & FGD417-780-Hypothetical protein & DY588293 \\
\hline
\end{tabular}


Table 1 (continued)

\begin{tabular}{lccc}
\hline ESTs found in the published $F$. graminearum genome sequence & $\begin{array}{l}\text { GenBank } \\
\text { accession } \\
\text { number }\end{array}$ \\
\hline Conditions $^{\mathrm{a}}$ & $\mathrm{No}^{\mathrm{b}}$ & FG ORF DNA $^{\mathrm{c}}$ & DY588294 \\
\hline B6/C6 & 2 & $\begin{array}{l}\text { FG10589-ext-Hypothetical protein, a possible joining to the conservative hypothetical } \\
\text { protein FG10590 }\end{array}$ & FG06416-ext-A possible extension
\end{tabular}

ESTs not found in the published $F$. graminearum genome sequence

$\begin{array}{llll}\text { A3/B6/C6 } & 5 & \text { Malonyl-CoA decarboxylase. (MagCon[0319a]), E-140 } & \text { DW177368 } \\ \text { B6 } & 1 & \text { Unknown } & \text { DW177369 } \\ \text { C6 } & 3 & \text { Unknown } & \text { DW177371 } \\ \text { C6 } & 3 & \text { Unknown } & \text { DW177372 } \\ \text { C6 } & 2 & \text { Unknown } & \text { DW177374 } \\ \text { C6 } & 1 & \text { Gibberella moniliformis cDNA clone(DR630210), 1E-07 } & \text { DW177375 }\end{array}$

${ }^{\mathrm{a}}$ Description of strain and culture conditions: $\mathrm{A}=$ F. graminearum 1194-15. $\mathrm{B}=$ F. graminearum 1104-14. $\mathrm{C}=F$. culmorum 1098-6. The number behind the letter is the number of weeks of cultivation on sterile rice (Fig. 1). The ESTs that were identified under all four ZON-producing conditions are marked 'All'.

${ }^{\mathrm{b}}$ The number of different oligo(dT) primers in combinations with the same arbitrary primer, giving the same DDRT-PCR product.

${ }^{\mathrm{c}}$ The ESTs are marked with the gene name according to the annotated MIPS FGDB if they are situated in the coordinates of the gene sequence, or with an additional -ext, e.g. FG01004-ext, if they are putative extensions of the predicted genes. Genes marked with 'No ORF hit' are located completely outside the MIPS gene annotations. The 'No ORF hits' are described with BLAST results (Cogeme, NCBI), with Cogeme Unisequence ID or GenBank Accession number for the best match in parentheses. E-values greater than E-05 are listed as 'Unknown'.

more scarce and when the fungus may be undergoing significantly more catabolism. These ESTs may not be involved in ZON production, even if they are expressed at times when $\mathrm{ZON}$ genes are expressed and when $\mathrm{ZON}$ is produced.

The up-regulated ESTs were classified into several functional categories, according to the MIPS FunCat (Ruepp et al. 2004). Most belonged to the five MIPS categories: Unclassified proteins (38\%), Metabolism $(15 \%)$; Proteins with binding function (10\%); Biogenesis of cellular components $(6.5 \%)$ and Cell rescue, defence and virulence (5.5\%) (Fig. 2). The others were distributed among 10 other MIPS categories (25\%).

Real-time gene expression of selected genes

In a previous study we found that genes in the ZON biosynthetic cluster are expressed between 4 and 14 days growth on sterile rice, with peak expression at 7 and 11 days depending on the strain studied (Lysoe et al., 2008, submitted). The expression levels of the four known ZON genes showed a normal bell-shaped pattern
Fig. 2 Functional distribution of genes up-regulated during ZON production in $F$. graminearum and $F$. culmorum according to MIPS FunCat (Ruepp et al. 2004)

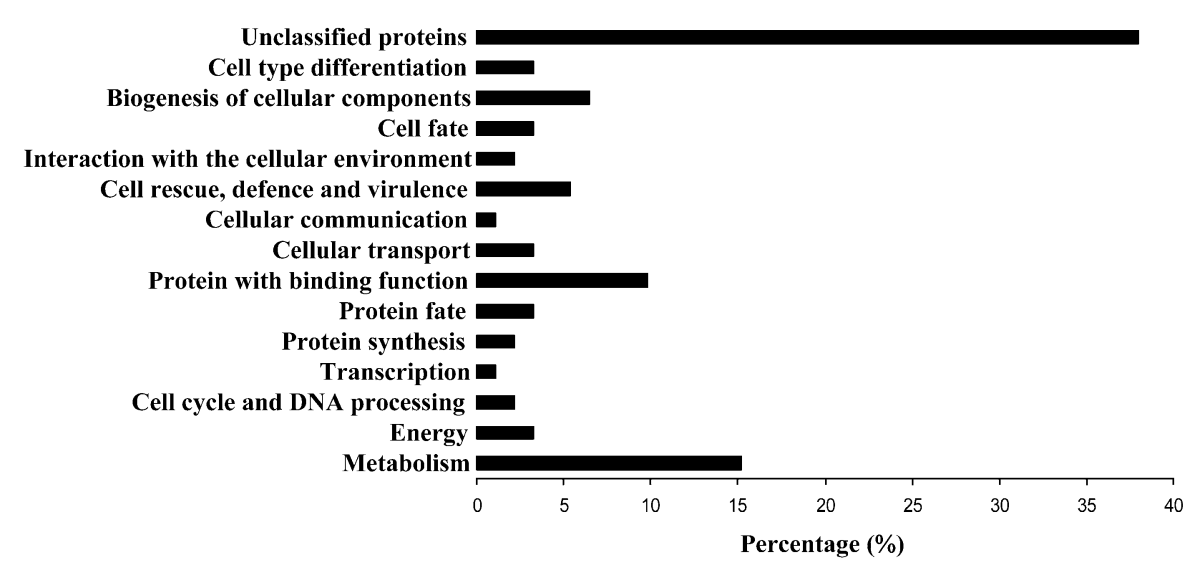


over time, with an increase in expression again at 14 days. Times later than 14 days were not studied, but the expression levels showed tendencies to a cyclic wavelike pattern. This cyclic pattern is also visualized in the amount of ZON produced in F. graminearum 110414 in Fig. 1, where ZON seem to be degraded or modified over time and then produced again.

We studied a subset of the DDRT-PCR ESTs, chosen on the basis of putative function and grouping in the genome, to examine if they were up-regulated under the same conditions. This was done by real-time qPCR to see if they followed the same expression patterns as genes in the ZON biosynthetic cluster, and to observe if these genes were down-regulated in our $\mathrm{ZON}$ negative $\triangle$ PKS4-T9 mutant compared to the wild-type strains (Fig. 3). Several of these genes were highly expressed under ZON-producing conditions, including genes similar to the hypothetical protein (FG10084), the calcium-binding EF-hand (FG05929), the septin aspE (FG08710), the histone deacetylase HOS3 (FG05636), the linoleate diol synthase (FG02668), the pisatin demethylase cytochrome P450 (FG03086), the hypothetical protein (FG03184), the gibberellin 20-oxidase (FG09103), the conserved hypothetical protein (FG01101) and the conserved hypothetical protein (FG05061). Genes with similarity to a $\mathrm{ABC1}$ transport protein (FG03735) and a hypothetical protein (FG09355) were expressed at low levels during these time points. None of these genes showed any up- or down-regulation in $\triangle \mathrm{PKS} 4-\mathrm{T} 9$ compared to the wildtype strains, except for the two genes FG02668 and FG03086 where the mutant showed a higher gene expression at 4 days growth. The expression patterns of up-regulated genes were not similar to those of $\mathrm{ZON}$ biosynthetic cluster genes and were not correlated with ZON accumulation (Fig. 4) as genes in the ZON biosynthetic cluster did (Lysoe et al., 2008, submitted). The ESTs from the DDRT-PCR study were found to be up-regulated at later time points on sterile rice (Table 1). The reason that they show early expression here is most likely because of the sensitivity of the real-time qPCR method compared to DDRT-PCR. The expression levels at these later time points could therefore possibly be higher than reported here.

Technical analysis of ESTs

We compared all 133 ESTs from both $F$. graminearum and F. culmorum to the annotated MIPS FGDB with the intention of improving some of the computational gene predictions by comparison with cDNA sequences. These ESTs are described with sequence coordinates, exon-intron boundaries and polyadenylation tails (when known). We found that 118 ESTs had a significant sequence match with the $F$. graminearum genome sequence (Table S2), and of these, 47 were situated inside the MIPS gene coordinates, 45 were situated both inside and outside (putative extensions) and 23 were completely outside the predicted genes. Several introns were observed in the ESTs. We could verify 10 of the MIPS intron predictions and also present 16 putative incorrect MIPS intron annotations that were in conflict with our data. In one case, we could see alternative splicing in MIPS gene FG05061, where both the presence (DY588264) of an intron could be found. In one singleton EST from $F$. graminearum (DY763108) we found evidence for a very long $(\sim 13,476 \mathrm{bp})$ missing gap. The other $18 \mathrm{ESTs}$, two from $F$. graminearum and 16 from $F$. culmorum, were not found in the published genome sequence (Table S3).

\section{Discussion}

Several clustered genes in the biosynthetic pathway of ZON have previously been identified, the two polyketide synthase genes PKS4 and PKS13, and genes similar to isoamyl alcohol oxidase (FG12056) and a regulatory protein (FG02398) (Gaffoor and Trail 2006; Kim et al. 2005; Lysoe et al. 2006). In an effort to identify additional genes that could somehow be related to ZON production, we performed DDRTPCR analysis on two F. graminearum strains and one F. culmorum strain that produced high amounts of ZON when grown on sterile rice. We identified 54 upregulated ESTs from samples corresponding to high ZON production, and did BLAST searches to reveal their putative gene function and their location in the $F$. graminearum genome sequence. None of the genes found in this study were located close to the ZON contigs $(1.118,1.119$ and 1.120$)$, but biosynthetic genes for fungal secondary metabolites could be found at multiple loci. For example trichothecene biosynthetic genes are located at four different loci (Desjardins 2006).

It has previously been reported that temperature stress can act as an enhancer for ZON production (Jimenez et al. 1996), but according to our results this 


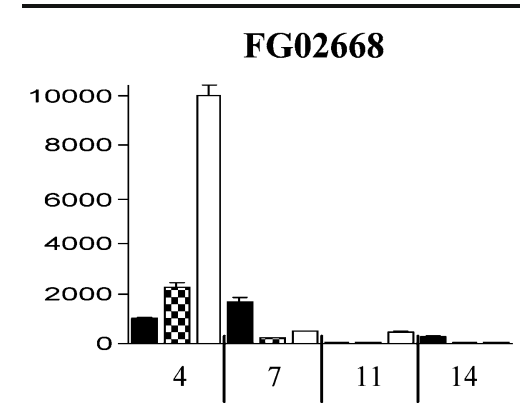

FG03184

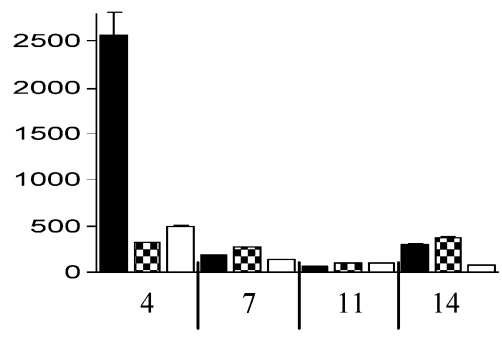

FG05929

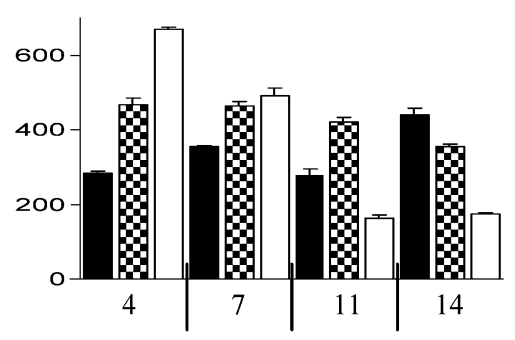

FG09103
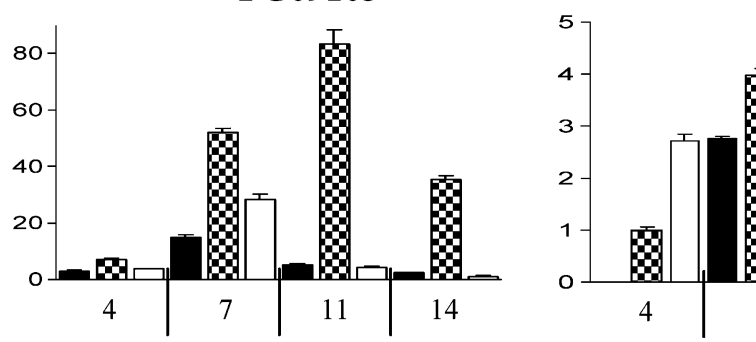

Fig. 3 Relative gene expression of some selected DDRT-PCR ESTs in the F. graminearum strains 1104-14 (black), 1166-9 (checker square) and the ZON negative mutant $\triangle \mathrm{PKS} 4-\mathrm{T} 9$ (white) after $4,7,11$ and 14 days growth on sterile rice at $25^{\circ} \mathrm{C}$

effect seems to be strain-dependent. The two $F$. graminearum isolates responded differently to the change in temperature from $25^{\circ} \mathrm{C}$ to $12^{\circ} \mathrm{C}$. Isolate 1104-14 showed a dramatic decrease in ZON production whereas 1194-15 increased its production. The $F$. culmorum isolate did not seem to respond to the temperature change. ZON usually accumulates during growth and is a stable compound in food and feed
FG03086

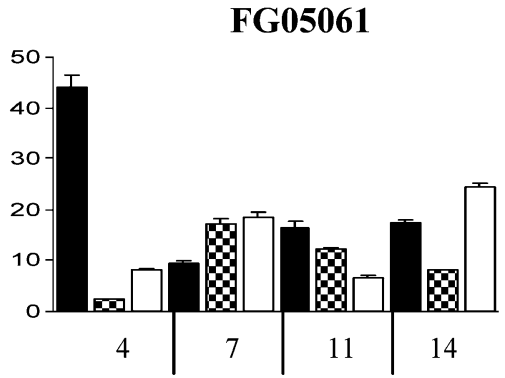

FG10084

FG03735

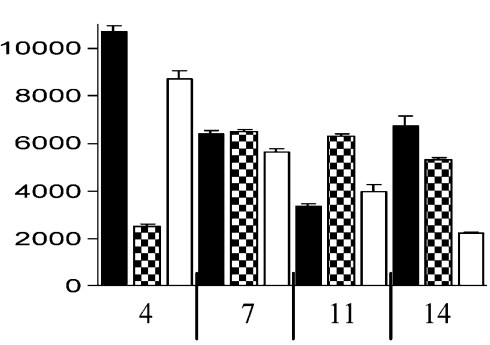

FG05636

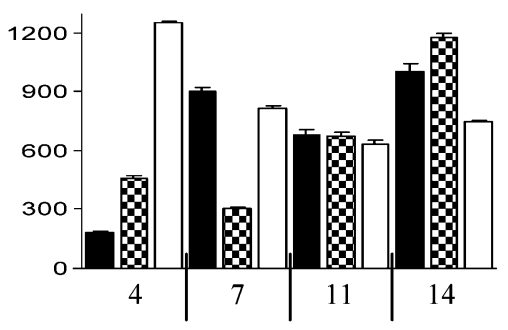

FG01101

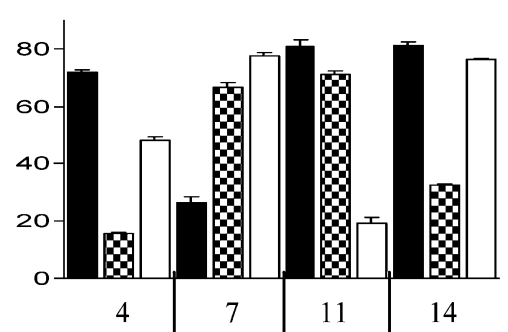

in the dark. $X$-axis: Days growth. $Y$-axis: Relative gene expression. The expression levels are relative to the alcohol oxidase gene FG12056 in strain 1104-14 after 7 days growth. Error bars indicate standard deviations of three replicates

(Lauren and Smith 2001). The observed reduction in ZON after the temperature shift might be due to a modification of $\mathrm{ZON}$ to a $\mathrm{ZON}$ derivative or because ZON was degraded by the fungus itself during growth. The ELISA kit employed in this study used monoclonal antibodies, and was not designed to detect the many different ZON derivatives (Shier et al. 2001), so any derivative or degradation product 


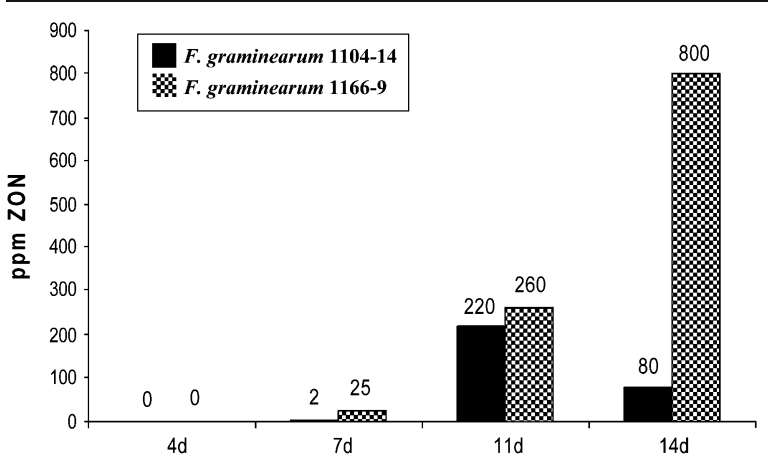

Fig. 4 HPLC analysis of the amount of ZON produced in $F$. graminearum 1104-14 and 1166-9 after 4, 7, 11 and 14 days growth on sterile rice. The numbers over the bars are the ppm ZON produced. The mutant $\triangle \mathrm{PKS} 4-\mathrm{T} 9$ showed no ZON production at any of the time points

would not have been detected. In another experiment, the expression levels of the four known $\mathrm{ZON}$ genes showed tendencies to a cyclic wavelike pattern over time when grown on sterile rice at constant $25^{\circ} \mathrm{C}$ (Lysoe et al., 2008, submitted). This could indicate that $\mathrm{ZON}$ is first produced, and after a pause, with no or low ZON synthesis, produced again. The observed reduction in ZON, especially at the DDRT-PCR experiments in strain 1104-14, could therefore possibly be explained by degradation or modification of $\mathrm{ZON}$ by the fungus because the levels had become too high for the fungus to thrive, and not as a result of the temperature shift. Concentrations around $1,000 \mathrm{ppm}$ in the media is considerable, and in perspective, 10,000 times higher than the maximum allowable ZON levels suggested by EU $(0.1 \mathrm{ppm})$ in unprocessed cereals other than maize.

Several of the up-regulated DDRT-PCR ESTs found in this study were located close together in the same contigs. Such clustering might therefore indicate that the corresponding genes are parts of the same biosynthetic pathway, or only that they accidentally are up-regulated at the same time. Members from six such putative pathways were found. The gene FG05928 was found together with the adjacent gene FG05929, with similarity to a calcium-binding EFhand with a putative regulatory function. The three genes FG02666 (similar to acetylxylan esterase precursor), FG02667 (conserved hypothetical protein) and FG02668 (similar to linoleate diol synthase) could encode putative proteins in a lipid, fatty acid and isoprenoid biosynthetic pathway in contig 1.132. Isoprenoids are primary metabolites known to be precursors of trichothecene biosynthesis. It has been shown that oxylipins, including hydroperoxylinoleic acid derivates of linoleic acid, metabolites of linoleate diol synthase, are involved in many signalling pathways in fungi, including as elicitors of sporulation in Aspergillus spp. (Tsitsigiannis et al. 2005). A gene similar to linoleate diol synthase has also been found up-regulated under conidiation and fumonisin biosynthesis in Fusarium verticillioides (Shim and Woloshuk 2001), and its products have been found to variously activate or inhibit aflatoxin synthesis and sexual sporulation in Aspergillus (Calvo et al. 2002; Champe and Elzayat 1989). A gene similar to linoleate diol synthase is also up-regulated under cercosporin production, a polyketide phytotoxin produced by Cercospora zeae-maydis (Shim and Dunkle 2002). Development of a knock-out mutant of FG02668 could therefore be interesting when studying mycotoxin production, signalling and sporulation in $F$. graminearum. The gene similar to gibberellin 20-oxidase (FG09103) was also up-regulated in the DDRT-PCR experiments. Gibberellins, like other diterpenoids, are produced from HMG coenzyme A, which converts HMG-CoA to mevalonate and is a key enzyme of the mevalonate pathway involved in the synthesis of many primary and secondary metabolites, such as sterols, carotenoids, trichothecenes, and gibberellins (Woitek et al. 1997). Two other genes located near each other, similar to an esterase (FG03081) and pisatin demethylase (FG03086), were up-regulated during DDRT-PCR. Pisatin demethylase detoxifies pisatin, and studies have shown that this could be a virulence factor in some plant pathogenic fungi (Delserone et al. 1999). The three up-regulated genes FG03184 (hypothetical protein), FG03190 (hypothetical protein) and FG03199 (similar to phenol 2-monooxygenase) were also found relatively close together on two contigs, 1.148 and 1.149. Monooxygenases are frequently found in secondary metabolite clusters.

Representatives from four of the putative gene clusters identified in this study, the genes similar to calcium-binding EF-hand (FG05929), linoleate diol synthase (FG02668), pisatin demethylase (FG03086), a hypothetical protein (FG03184), and gibberellin 20oxidase (FG09103), showed high expression during some of the time points in the two wild-type strains 1104-14 and 1166-9 in our real-time qPCR study. The putative $\mathrm{ABC} 1$ transport protein gene $\mathrm{FG} 03735$ was found close to a putative esterase gene (FG03738) in 
contig 1.162, but real-time qPCR showed that the expression of FG03735 in the two wild-types 1104-14 and 1166-9 was low. The last two genes identified by DDRT-PCR that might be involved in the same pathway, the FG07075 (similar to PYC2-pyruvate carboxylase 2) and the FG07076 (similar to cysteine-rich protein NFX-1), were not analyzed by real-time qPCR.

Some other up-regulated DDRT-PCR genes were also analyzed by real-time qPCR, showing high expression during some of the time points in the two wild-type strains 1104-14 and 1166-9. These were the genes with similarity to the hypothetical protein (FG10084), the septin aspE (FG08710), the histone deacetylase HOS3 (FG05636), the conserved hypothetical protein (FG01101) with a TPR-like domain and the conserved hypothetical protein (FG05061). The hypothetical protein (FG10084) was identified by similarity search to be a transmembrane glycoprotein containing a cytochrome P450 domain, having resemblance to several proteins in the trichothecene mycotoxin pathway. It also contained a von Willebrand type A ATPase domain similar to those that bind collagen and platelets in blood clotting mechanisms. Being situated in a membrane and having both a binding and an oxygenase capacity, this protein could be part of a detox pump or transporter. The gene similar to septin aspE (FG08710) shows resemblance to the aspE gene from Aspergillus nidulans. Members of the septin gene family are often involved in cytokinesis and the organization of new growth. The FG05636 shows strong similarity to HOS3, an HDAC involved in chromatin remodelling and gene expression, so it could be involved in regional or global regulation. It would be interesting to see how a knockout of this gene would influence ZON production. The FG01101 protein sequence seems to have a TPR motif, usually involved in protein-protein interactions in a variety of biological processes, such as cell cycle regulation, transcriptional control, mitochondrial and peroxisomal protein transport, neurogenesis and protein folding. The FG05061 protein sequence has some similarity to a mating type 1 pheromone precursor, which could be involved in female and male behaviour in filamentous ascomycete fungi.

The enzymatic mechanisms of polyketide and fatty acid synthesis are similar, and genes for the biosynthesis of fatty acids are also found in secondary metabolite clusters, e.g. in the biosynthesis of aflatoxin (Yu et al. 2004). A comparative genomic analysis of phytopathogenic fungi using EST collections (COGEME) shows that there are significant differences in the expressed gene inventories between the genomes of pathogens and non-pathogens, with groups of genes encoding products involved in lipid metabolism, for instance, being more highly represented in expressed gene collections from pathogenic species (Soanes and Talbot 2006). A secreted lipase in F. graminearum is also a virulence factor during infection of cereals (Voigt et al. 2005). Genes encoding proteins commonly found associated with secondary metabolite production or lipid metabolism were in this study found to be up-regulated during ZON production, e.g. dehydrogenase, esterase, oxidoreductase, monooxygenase, cytochrome P450 and a linoleate diol synthase. Further characterization of these genes should therefore aid in identifying the interrelationships of these pathways.

The real-time gene expression study verified that the transcription of several DDRT-PCR ESTs was upregulated in the two wild-type strains during $\mathrm{ZON}$ production, but no up- or down-regulation in the $\mathrm{ZON}$ negative $\triangle$ PKS4-T9 could be found, except for the two genes FG02668 and FG03086 which were upregulated in $\triangle \mathrm{PKS} 4-\mathrm{T} 9$ after 4 days growth. The expression of the genes showed neither high similarity to the expression patterns of previously known ZON-related genes (Lysoe et al., 2008, submitted), nor did they follow the patterns of ZON accumulation. This implies that these genes are partially or fully under the control of other regulatory systems. This finding is not surprising, as genes involved in isoprenoid and lipid metabolism, for example, though obviously selectively active during ZON production, are also involved in primary metabolism. As there are two known signal transduction pathways and a chromatin remodelling mechanism in filamentous fungi that act globally to influence secondary metabolite gene clusters (Yu and Keller 2005), more work should be directed towards elucidating these complex interactions. The genes from our real-time expression study might not be directly involved in $\mathrm{ZON}$ biosynthesis, but could still be indirectly involved in ZON-related processes.

Fusarium culmorum is a common species in northern Europe (Kosiak et al. 2003), and is related to the nine phylogenetic lineages now recognized within the $F$. graminearum species complex (O'Donnell et al. 2004). Comparison of our EST sequences to the $F$. 
graminearum genome sequence revealed that $97 \%$ of the $F$. graminearum and $72 \%$ of the $F$. culmorum ESTs showed significant alignments. The remaining $F$. graminearum ESTs with no similarity found apparently fall within the sequence gaps in the genome sequence, or represent genes unique to the strains used to produce the cDNA. The $F$. graminearum Affymetrix GeneChip detects $98 \%$ of $F$. graminearum and $85 \%$ of $F$. culmorum DNA, but only $8 \%$ of $F$. oxysporum (Guldener et al. 2006b). The similarity of our $F$. culmorum ESTs to the $F$. graminearum genome sequence is somewhat lower than the Affymetrix GeneChip detection, but it gives an indication of the relationship between these two species.

Gene prediction programmes perform best on 'internal' exons, and not very well on initial and terminal exons or exons that correspond to the $5^{\prime}$ and 3' untranslated regions (UTRs) of mRNA (Guigo et al. 2000). About $50 \%$ of our ESTs with similarity to the annotated MIPS genes aligned to sequences both inside and outside of the MIPS genes. This could be due to real gene extensions or ESTs extending into the untranslated regions in the mRNA. In the work with the genome sequence of Caenorhabditis elegans, more than $90 \%$ of the genes have been directly supported by experimental evidence. Hillier et al. (2005) showed that the accuracy of individual exon prediction was high but the prediction of complete genes was less reliable, because of the combinatorics of multiexon genes and the challenge in detecting start and stop codons of genes. Of all our ESTs, 27\% are presented with the putative 3 ' end polyadenylation tail (marked PA in Table S2), but multiple polyadenylation sites could exist for the same gene. Several introns were also found, and we could confirm 10 MIPS introns and showed that 16 ESTs were in conflict with MIPS intron annotation.

In conclusion, we found several genes that were up-regulated when Fusarium spp. produced the estrogenic mycotoxin ZON. Four genes in the ZON cluster have already been characterized, and this study was an attempt to identify additional putative ZONrelated genes, and to study the effect of high $\mathrm{ZON}$ production on gene expression patterns of other genes in F. graminearum and F. culmorum. A number of the ESTs described here could be associated with secondary metabolite production and lipid metabolism, or could be involved in transcriptional activation, provision of precursors, signalling, transport or detoxification. Several of the ESTs were also situated close together in the same contigs and the information obtained here could be useful in the process of linking specific genes to biosynthetic pathways. Our work also contributes to the ongoing gene validation process for this important plant pathogenic fungus.

Acknowledgements This work was carried out at the Plant Health and Plant Protection Division, Bioforsk-Norwegian Institute for Agricultural and Environmental Research, Ås, Norway, as part of Erik Lysøe's Ph.D. thesis. Thanks to Dr. Barbara Kosiak and Dr. Trude Vrålstad of the National Veterinary Institute, Norway, for providing fungal strains. Grants from the Research Council of Norway (147003/130) funded this work.

\section{References}

Bottalico, A., \& Perrone, G. (2002). Toxigenic Fusarium species and mycotoxins associated with head blight in small-grain cereals in Europe. European Journal of Plant Pathology, 108, 611-624.

Calvo, A. M., Wilson, R. A., Bok, J. W., \& Keller, N. P. (2002). Relationship between secondary metabolism and fungal development. Microbiology and Molecular Biology Reviews, 66, 447-459.

Champe, S. P., \& Elzayat, A. A. E. (1989). Isolation of a sexual sporulation hormone from Aspergillus nidulans. Journal of Bacteriology, 171, 3982-3988.

Coulombe, R. A. (1993). Biological action of mycotoxins. Journal of Dairy Science, 76, 880-891.

Delserone, L. M., McCluskey, K., Matthews, D. E., \& Vanetten, H. D. (1999). Pisatin demethylation by fungal pathogens and nonpathogens of pea: Association with pisatin tolerance and virulence. Physiological and Molecular Plant Pathology, 55, 317-326.

Desjardins, A. E. (2006). In A. E. Desjardins (Ed.), Fusarium mycotoxins: Chemistry, genetics and biology p. 260. St. Paul, Minnesota, U.S.A.: APS Press.

Gaffoor, I., Brown, D. W., Plattner, R., Proctor, R. H., Qi, W. H., \& Trail, F. (2005). Functional analysis of the polyketide synthase genes in the filamentous fungus Gibberella zeae (anamorph Fusarium graminearum). Eukaryotic Cell, 4, 1926-1933.

Gaffoor, I., \& Trail, F. (2006). Characterization of two polyketide synthase genes involved in zearalenone biosynthesis in Gibberella zeae. Applied and Environmental Microbiology, 72, 1793-1799.

Guigo, R., Agarwal, P., Abril, J. F., Burset, M., \& Fickett, J. W. (2000). An assessment of gene prediction accuracy in large DNA sequences. Genome Research, 10, 1631-1642.

Guldener, U., Mannhaupt, G., Munsterkotter, M., Haase, D., Oesterheld, M., Stumpflen, V., et al. (2006a). FGDB: A comprehensive fungal genome resource on the plant pathogen Fusarium graminearum. Nucleic Acids Research, 34, 456-458.

Guldener, U., Seong, K. Y., Boddu, J., Cho, S., Trail, F., Xu, J. R., et al. (2006b). Development of a Fusarium 
graminearum Affymetrix GeneChip for profiling fungal gene expression in vitro and in planta. Fungal Genetics and Biology, 43, 316-325.

Hillier, L. W., Coulson, A., Murray, J. I., Bao, Z. R., Sulston, J. E., \& Waterston, R. H. (2005). Genomics in C. elegans: So many genes, such a little worm. Genome Research, 15, 1651-1660.

Jimenez, M., Manez, M., \& Hernandez, E. (1996). Influence of water activity and temperature on the production of zearalenone in corn by three Fusarium species. International Journal of Food Microbiology, 29, 417-421.

Kim, Y. T., Lee, Y. R., Jin, J. M., Han, K. H., Kim, H., Kim, J. C., et al. (2005). Two different polyketide synthase genes are required for synthesis of zearalenone in Gibberella zeae. Molecular Microbiology, 58, 1102-1113.

Kosiak, B., Torp, M., Skjerve, E., \& Thrane, U. (2003). The prevalence and distribution of Fusarium species in Norwegian cereals: A survey. Acta Agriculturae Scandinavica Section B-Soil and Plant Science, 53, 168-176.

Langseth, W., Bernhoft, A., Rundberget, T., Kosiak, B., \& Gareis, M. (1998). Mycotoxin production and cytotoxicity of Fusarium strains isolated from Norwegian cereals. Mycopathologia, 144, 103-113.

Lauren, D. R., \& Smith, W. A. (2001). Stability of the Fusarium mycotoxins nivalenol, deoxynivalenol and zearalenone in ground maize under typical cooking environments. Food Additives and Contaminants, 18, 1011-1016.

Lysoe, E., Klemsdal, S. S., Bone, K. R., Frandsen, R. J. N., Johansen, T., Thrane, U., et al. (2006). The PKS4 gene of Fusarium graminearum is essential for zearalenone production. Applied and Environmental Microbiology, 72, 3924-3932.

O’Donnell, K., Ward, T. J., Geiser, D. M., Kistler, H. C., \& Aoki, T. (2004). Genealogical concordance between the mating type locus and seven other nuclear genes supports formal recognition of nine phylogenetically distinct species within the Fusarium graminearum clade. Fungal Genetics and Biology, 41, 600-623.

Parry, D. W., Jenkinson, P., \& Mcleod, L. (1995). Fusarium ear blight (scab) in small-grain cereals-A review. Plant Pathology, 44, 207-238.

Pfaffl, M. W., Horgan, G. W., \& Dempfle, L. (2002). Relative expression software tool (REST (c)) for group-wise comparison and statistical analysis of relative expression results in real-time PCR. Nucleic Acids Research, 30(9), e36.

Pfaffl, M. W., Tichopad, A., Prgomet, C., \& Neuvians, T. P. (2004). Determination of stable housekeeping genes, differentially regulated target genes and sample integrity:
BestKeeper-Excel-based tool using pair-wise correlations. Biotechnology Letters, 26, 509-515.

Reischer, G. H., Lemmens, M., Farnleitner, A., Adler, A., \& Mach, R. L. (2004). Quantification of Fusarium graminearum in infected wheat by species specific real-time PCR applying a TaqMan Probe. Journal of Microbiological Methods, 59, 141-146.

Ruepp, A., Zollner, A., Maier, D., Albermann, K., Hani, J., Mokrejs, M., et al. (2004). The FunCat, a functional annotation scheme for systematic classification of proteins from whole genomes. Nucleic Acids Research, 32, 55395545.

Shier, W. T., Shier, A. C., Xie, W., \& Mirocha, C. J. (2001). Structure-activity relationships for human estrogenic activity in zearalenone mycotoxins. Toxicon, 39, 1435-1438.

Shim, W. B., \& Dunkle, L. D. (2002). Identification of genes expressed during cercosporin biosynthesis in Cercospora zeae-maydis. Physiological and Molecular Plant Pathology, 61, 237-248.

Shim, W. B., \& Woloshuk, C. P. (2001). Regulation of fumonisin B-1 biosynthesis and conidiation in Fusarium verticillioides by a cyclin-like (C-type) gene, FCC1. Applied and Environmental Microbiology, 67, 1607-1612.

Smedsgaard, J. (1997). Micro-scale extraction procedure for standardized screening of fungal metabolite production in cultures. Journal of Chromatography A, 760, 264-270.

Soanes, D. M., \& Talbot, N. J. (2006). Comparative genomic analysis of phytopathogenic fungi using expressed sequence tag (EST) collections. Molecular Plant Pathology, 7, 61-70.

Tsitsigiannis, D. I., Kowieski, T. M., Zarnowski, R., \& Keller, N. P. (2005). Three putative oxylipin biosynthetic genes integrate sexual and asexual development in Aspergillus nidulans. Microbiology, 151, 1809-1821.

Voigt, C. A., Schafer, W., \& Salomon, S. (2005). A secreted lipase of Fusarium graminearum is a virulence factor required for infection of cereals. Plant Journal, 42, 364-375.

Woitek, S., Unkles, S. E., Kinghorn, J. R., \& Tudzynski, B. (1997). 3-Hydroxy-3-methylglutaryl-CoA reductase gene of Gibberella fujikuroi: Isolation and characterization. Current Genetics, 31, 38-47.

Yu, J. J., Chang, P. K., Ehrlich, K. C., Cary, J. W., Bhatnagar, D., Cleveland, T. E., et al. (2004). Clustered pathway genes in aflatoxin biosynthesis. Applied and Environmental Microbiology, 70, 1253-1262.

Yu, J. H., \& Keller, N. (2005). Regulation of secondary metabolism in filamentous fungi. Annual Review of Phytopathology, 43, 437-458. 